\title{
A natural orbital branching scheme for time dependent density functional theory nonadiabatic simulations
}

\author{
Lin-Wang Wang ${ }^{1}$ \\ ${ }^{1}$ Materials Sciences Division, Lawrence Berkeley \\ National Laboratory, Berkeley, California 94720, USA
}

(Dated: July 12, 2020)

\begin{abstract}
Real time time dependent density functional theory (rt-TDDFT) has now been used to study a wide range of problems, from optical excitation, to charge transfer, to ion collision, to ultrafast phase transition. However, conventional rt-TDDFT Ehrenfest dynamics for nuclear movement lacks a few critical features to describe many problems: the detail balance between state transition, decoherence for the wave function evolution, and stochastic branching of the nuclear trajectory. There are many-body formalisms to describe such nonadiabatic molecular dynamics, especially the ones based on mixed quantum/classical simulations, like the surface hopping and wave function collapsing schemes. However, there are still challenges to implement such many-body formalisms to the rt-TDDFT simulations, especially for large systems where the excited state electronic structure configuration space is large. Here we introduce two new algorithm for nonadiabatic rt-TDDFT simulations: the first is a Boltzmann factor algorithm which introduces decoherence and detailed balance in the carrier dynamics, but uses mean field theory for nuclear trajectory. The second is a natural orbital branching (NOB) formalism, which use time dependent density matrix for electron evolution, and natural orbital to collapse the wave function upon. It provides decoherence, detailed balance and trajectory branching properties. We have tested these methods for a molecule radiolysis decay problem. We found these methods can be used to study such radiolysis problem in which the molecule is broken into many fragments following complex electronic structure transition paths. The computational time of NOB is similar to the original plain rt-TDDFT simulations.
\end{abstract}




\section{INTRODUCTION}

Time dependent evolution of the Schrodinger's equation has a history as old as the quantum mechanics itself ${ }^{1,2}$. While ground state calculations yield atomic structures and binding energies, time dependent solution of the Schrodinger's equation reveals the dynamics of the system. Much like in the ground state calculation, the time dependent solution of the Schrodinger's equation is often based on Born-Oppenheimer expansion of the electronnuclear wave functions, taking the advantage of the large mass difference between electron and nuclei ${ }^{3}$. In case where the nuclear movement needs to be described fully quantum mechanically, one can first get the potential energy surface (PES) $E_{k}(\{R\})$ of the nuclear degree of freedom $\{R\}$ by solving the many-body electronic adiabatic eigen state $\Psi_{k}(r, R)$ (where $E_{k}(\{R\})$ is its eigen energy) at every nuclear atomic configuration $\{R\}$, where $k$ is an index of the electronic configuration. We can then solve the nuclear time dependent Schrodinger's equation based on the PES $E_{k}(\{R\})$ including the transition between different $\mathrm{k}^{\prime}{ }^{4}$. In practice, it is only possible to precalculate the full $E_{k}(\{R\})$ for small molecules, and considering only a limited electronic configurations $\mathrm{k}^{4}$.

To analyze the dynamics process, one can express the total electron nuclear wave function $\Omega(r, R, t)$ in the Born-Huang expansion ${ }^{3}: \sum_{k} C_{k}(t) \Psi_{k}(r, R) \Phi_{k}(R, t)$ where $\Psi_{k}(r, R)$ does not depend on the time t. One can then write down the time dependent equation of $\Phi_{k}(R, t)$ based on the PES of $\Psi_{k}(r, R)$, and the coupling between $\mathrm{k}$ and $\mathrm{k}^{\prime}:\left\langle\Psi_{k}(r, R)\left|\frac{\partial}{\partial R}\right| \Psi_{k^{\prime}}(r, R)>\right.$ (here $\mathrm{R}$ acts as a parameter). Such coupling introduces quantum transition flux between different adiabatic states $\mathrm{k}^{5}$. One approximation is to use Gaussian wave packets to represent the nuclear wave function $\Phi_{k}(R, t)^{6}$. In the full multiple spawning (FMS) $\operatorname{method}^{7}$, the $\Phi_{k}(R, t)$ is represented by a number of Gaussian functions with fixed widths, while the trajectory $R_{n}(t)$ (subscribe $\mathrm{n}$ is an index of the trajectory) of the center of the Gaussian function follows the Newton's law on the PES of the corresponding electronic state. A trajectory $R_{n}(t)$ can be spawned into two at $\mathrm{R}$ where the amplitude of $<\Psi_{k}(r, R)\left|\frac{\partial}{\partial R}\right| \Psi_{k^{\prime}}(r, R)>$ is large. Then a child trajectory residing on the electronic state k' can be generated ${ }^{7}$. Since the $\Psi_{k}(r, R)$ related to a given trajectory $R_{n}(t)$ can be approximated by $\Psi_{k}\left(r, R_{n}(t)\right)$, it can be calculated on-the-fly, making the calculation much cheaper than the full PES based quantum calculations. In an alternative approach, a nuclear trajectory $R(t)$ does not spawn into two, but it can hop from one k PES to another k' PES, using $<\Psi_{k}(r, R(t))\left|\frac{\partial}{\partial R}\right| \Psi_{k^{\prime}}(r, R(t))>\cdot \dot{R}(t)$ 
as a guide for the hopping probability ${ }^{8}$. An assemble of trajectories are used to get the statistical properties. This is the surface hopping ( $\mathrm{SH})$ scheme $^{8}$.

The above schemes use quantum mechanical description for the electron wave function and classical trajectory for nuclear movement, they are thus called mixed quantum/classical methods. In such methods, $\Omega(r, R, t) \doteq \sum_{k} C_{k}(t) \Psi_{k}(r, R(t)) \Phi_{k}(R, t)$. Suppose at $t=0$, we only have one $\Psi_{k}(r, R(t)) \Phi_{k}(R, t)$ term. If we focus on the time evolution of the electron wave function, then due to interstate coupling, this one term can be split into two terms: $\Psi_{k}(r, R(t)) \Phi_{k}(R, t)$ and $\Psi_{k^{\prime}}(r, R(t)) \Phi_{k^{\prime}}(R, t)$. Immediately after this electron wave function splitting, although $\Psi_{k^{\prime}}(r, R(t))$ is different (orthogonal) to $\Psi_{k}(r, R(t)), \Phi_{k^{\prime}}(R, t)$ is the same as $\Phi_{k}(R, t)$ (one can view $\Phi_{k}$ as ridding on the splitting of $\Psi_{k}$ ). However, due to their subsequent trajectory difference, $<\Phi_{k}(R, t) \mid \Phi_{k^{\prime}}(R, t)>$ will decay from one to zero exponentially with a time scale $\tau_{k, k^{\prime}}$. As a result, in terms of the electron wave function propagation, $\Psi_{k^{\prime}}(r, R(t))$ cannot be scattered back to interfere with $\Psi_{k}(r, R(t))$ (due to their ridding nuclear wave function $\Phi_{k}, \Phi_{k}^{\prime}$ differences). Thus, effectively, $\Omega(r, R, t)$ can be represented by an classical assembly of non interference wave functions $\Psi_{h}\left(r, R_{h}(t)\right)$. This is the basis of the wave function collapsing (WFC) scheme of the mixed quantum/classical approach, which focus more on the evolution of the electron wave function $\Psi(r, R(t))$. The sudden collapse of the electron wave function adds dissipative feature in the time dependent Schrodinger's equation for $\Psi(r, R(t))^{9}$. In this view, it is the decoherence which leads to wave function collapsing, which leads to the hopping of the trajectory and stochastic trajectory branching. Thus WFC also has the SH component in it. It is one way to realize SH. In terms of their derivations, the original $\mathrm{SH}$ is focused more on the nuclear wave function $\Phi_{k}(R, t)$, its evolution with time, and how it can branch into different $\mathrm{k}$ (thus trajectory hopping), while WFC is more focused on the time evolution of the electron wave function, and how different parts of the wave function lose coherence with each other due to the nuclear wave functions ridding on them ${ }^{5}$. The decoherence effect exhibits itself explicitly in the WFC scheme, while it needs to be introduced additionally in the pure $\mathrm{SH}$ approaches ${ }^{1}$.

The other effect which needs to be enforced is the detailed balance. In the time dependent Schrodinger's equation for the electron wave function $\Psi(r, t)$, there are transitions between adiabatic states $\Psi_{k}(r, R(t))$ and $\Psi_{k^{\prime}}(r, R(t))$. This transition does not depend on their potential energy difference: $d E_{k, k^{\prime}}=E_{k}(R(t))-E_{k^{\prime}}(R(t))$. In reality however, if the nuclear degree of freedom is also described quantum mechanically, and if finite temperature $\mathrm{T}$ is 
used to describe the occupation of the phonon wave function, then due to the existence of zero phonon mode, the efficiencies of the two transitions (from k to k', versus from k' to k) differ by an Boltzmann's factor $\exp \left(-d E_{k, k^{\prime}} / k T\right)$, which favor the transition from higher energy adiabatic state to lower energy adiabatic state. This is also called detailed balance, which is the cause for hot carrier cooling.

It is worth to mention that there are many other nonadiabatic molecular dynamics approaches based on mixed quantum/classical scheme, like: quantum/classical Liouville approach $^{10}$, Bohmian dynamics ${ }^{11}$ and exact-factorization scheme ${ }^{12}$.

The simplest mixed quantum/classical approach is the mean field Ehrenfest (MFE) dynamics ${ }^{13,14}$. However, since it does not have the decoherence and detailed balance effects $^{15-17}$, one has to introduce them explicitly either by deploying the $\mathrm{SH}$ scheme or WFC scheme. One of the most popular SH scheme is the fewest switches surface hopping (FSSH) approach ${ }^{8}$, where the nuclear trajectory is based on one potential energy surface PES $E_{k}(R)$ until it hopes to another PES $E_{k^{\prime}}(R)$ in a stochastic fashion depending on the coupling between $\Psi_{k}(r, R(t))$ and $\Psi_{k^{\prime}}(r, R(r))$. The original $\mathrm{FSSH}^{8}$ does not have decoherence, which can be introduced by modifying the coefficients of wave functions after every time step of the Schrodinger's equation ${ }^{18,19}$. The schemes with WFC components include: instantaneous decoherence ${ }^{20}, \mathrm{~A}-\mathrm{FSSH}^{21}$, decoherence induced surface hopping (DISH) ${ }^{22}$ and mean-field dynamics with stochastic decoherence (MF-SD) ${ }^{23}$. In such WFC schemes, a time dependent wave function $\Psi(r, t)=\sum C_{k} \Psi_{k}(r, t)$ will be stochastically collapse into a new wave function $\Psi^{\prime}(r, t)$ consisted with a subset of the adiabatic states $\Psi_{k}$, e.g, by setting one or few $C_{k}$ to zero. The time dependent Schrodinger's equation will restart from $\Psi^{\prime}(r, t)$. As mentioned before, such WFC scheme has also SH feature, since the WFC helps the system to jump into different PES. In both the pure SH and WFC schemes, immediately after the hopping or collapsing event, the total energy needs to be conserved. If the transition is from a lower energy to a higher energy state, the increased potential energy needs to be compensated by a kinetic energy reduction along a transition degree of freedom (TDF) (which is the coupling direction $\Psi_{k}(r, R(t))\left|\frac{\partial}{\partial R}\right| \Psi_{k^{\prime}}(r, R(t))>.{ }^{24}$ If this TDF does not have sufficient kinetic energy, the attemped transition will need to be cancelled. This introduces the required detailed balance in an approximated way.

The above mixed quantum/classical schemes are derived based on many-body wave functions, here $\Psi_{k}(r, R(t))$ is the many-body adiabatic state $\mathrm{k}, E_{k}(R(t))$ is its eigenenergy and 
$r \in R^{3 N_{e}}$, where $N_{e}$ is the number of electrons. There is a tremendous interest to deploy the above procedures to density functional theory $(\mathrm{DFT})^{25}$, where large systems (e.g., more than 100 atoms) can be calculated. The real-time time dependent density functional theory (rt-TDDFT $)^{26}$ has now been used to study a wide range of problems, including light absorption spectrum; ion collision stoping power; hot carrier dynamics; and laser pulse induced ultrafast phase changes etc. However, the rt-TDDFT is based on the mean field Ehrenfest dynamics, thus it lacks the decoherence, detailed balance and stochastic branching features. This makes it impossible to study some important physical phenomena, like the hot carrier cooling; molecule breaking by radiolysis; chemical reaction branching; and photochemistry. Without detailed balance, the Ehrenfest dynamics often over heats the electronic degree of freedom ${ }^{15-17}$. As a pure deterministic method, it cannot be used to estimate the probability of different products for a given chemical reaction.

There are many works in implementing the above many-body schemes under DFT framework ${ }^{5,22,27-31}$. There are however several challenges. In the wave function based quantum chemistry methods, the many-body wave function $\Psi_{k}(r, R(t))$ for which most of the above algorithms are based on is calculated explicitly. Within DFT, the $\Psi_{k}(r, R(t))$ does not exist, thus has to be approximated. A widely adopted approach ${ }^{30}$ for photochemistry problems is to use linear response TDDFT method to calculate the exciton states using Casida equation, and using Casida coefficient and the single excitation Slater determinant (SD) basis set to approximate the many body wave function $\Psi_{k}(r, R(t))$. Besides being relatively expensive, this approach cannot be used to study problems with more than a single electron excitations. One can used a self-consistent field (SCF) constraint DFT with a given occupation configuration $\mathrm{k}$ to calculate the excited state energy ${ }^{31}$. In this scheme, the SD of the given configuration $\mathrm{k}$ is used to represent the many-body wave function $\Psi_{k}(r, R(t))$. The many-body wave function time evolution equation is provided by the constraint DFT calculated PES and the state coupling calculated using the SD. Note, in this scheme, due to SCF calculation, different occupation configuration $\mathrm{k}$ will have different Kohn-Sham single particle orbital $\phi_{i, k}(r, R(t))$ (here unlike above, $r \in R^{3}$ is the single electron coordinate, and we use lower case Greek symbol to represent the single particle wave functions). As a result, the SD for different $\mathrm{k}$ might not be orthogonal to each other. An orthogonalization procedure is needed to fix this problem ${ }^{31}$. One can simplify this procedure as in the DISH $\operatorname{method}^{22,27,32}$, where the single particle time dependent Schrodinger's equation is used to 
evolve $\psi_{j}(r, t)$, much like in the conventional rt-TDDFT ${ }^{32}$. However, the $\psi_{j}(r, t)$ is expanded by the single particle adiabatic states $\phi_{i}(r, t)$ of the charge density $\rho(r, t)$ at time t. Thus the SD of $\left\{\psi_{j}(r, t)\right\}$ will be a linear combination of many SD of $\left\{\phi_{i}(r, t)\right\}$ for all the possible selections of $N_{e}$ adiabatic states i. Then the wave function will be collapsed into one or several SD of $\left\{\phi_{i}(r, t)\right\}$, which is used to approximate the many-body adiabatic states. Since a unified set of $\left\{\phi_{i}(r, t)\right\}$ is used, there is no orthogonalization problem as in the above constraint DFT approach. ${ }^{31}$. Besides, there is no need for a SCF calculation for each occupation configuration $\mathrm{k}$, thus the calculation is much faster. However, there could be issues for the PES of one SD of $\left\{\phi_{i}(r, t)\right\}$, since it is not selfconsistently calculated. Besides, immediately after the wave function collapsing, $\left\{\phi_{i}(r, t)\right\}$ are no longer single particle adiabatic eigen states for the after collapsing charge density $\rho^{\prime}(r, t)$, thus they are not stationary for time evolution, which makes it different from the original many-body based scheme.

In the actual implementation of the many-body wave function based schemes, one practical difficult is the large number of possible occupation configurations $k$. In the molecular quantum chemistry calculation, very often only a few excited states are considered. In the large system we are interested for rt-TDDFT calculations, and for strong multi-state excitations, the configuration space can grow exponentially with system size. Thus how to keep track of all these states becomes a computational challenge.

Lastly, it is worth to mention that it is still an open issue for how accurate can DFT/TDDFT describe the dynamics behavior of the excited state, for example to describe the intersection seams at the conical intersection, which is important for small molecule photochemistry $^{33}$. For example, it was found that, while the overall picture of the PES can be described well by linear response TDDFT ${ }^{29,34}$, there could be some small qualitative difference (degeneracy splitting) between the linear response TDDFT results and high order quantum chemistry results for small molecule single state excitations ${ }^{34}$. However, it is possible that for more complex systems, and stronger many state excitations, this might no longer be true, or the dynamics might not be so sensitively depend on the intersection seams. After all, in all the above algorithms, the trajectory hopping and wave function collapsing happen at $\mathrm{R}$ point where there is still an energy difference between the two PES (e.g., due to avoid crossing ${ }^{35}$ ).

Note that, for some problems, one can just solve the single particle time dependent Schrodinger's equation using one single particle time evolving wave function $\psi(r, t)$ without 
worrying about the many-body aspects. This can be used to simulate a single hot carrier cooling and carrier transport ignoring the possible carrier-carrier interaction, and the backreaction to the nuclear dynamics. Under classical path approximation (CPA) (which ignores the effect of electron to the nuclear dynamics backreaction), the ground state ab initio MD can be used to provide the single particle Kohn-Sham time dependent Hamiltonian $\mathrm{H}(\mathrm{t})$, on which the time dependent Schrodinger's equation $i \partial \psi(r, t) / \partial t=H(t) \psi(r, t)$ can be solved. There is also an decoherence and detailed balance issue since they are not in the $i \partial \psi(r, t) / \partial t=H(t) \psi(r, t)$ equation. The solution for this problem is simpler. For example, we have developed a P-matrix scheme ${ }^{36}$ where the problem is solved based on a density matrix, where the decoherence and detailed balance are introduced.

Overall, there are still challenges in implementing the many-body mixed quantum/classical schemes for nonadiabatic dynamics on DFT. It will be useful to explore alternative methods which fits more naturally to rt-TDDFT frameworks. In the current work, we introduce two new methods to describe the nonadiabatic MD within rt-TDDFT formalism. The first is a Boltzmann method, which incorporates the decoherence and detailed balance in the time dependent Schrodinger's equation, while it uses a mean field approach for nuclear dynamics. Unlike the CPA single particle nonadiabatic molecular dynamic approach ${ }^{36}$, in this Boltzmann-TDDFT method, the backreaction is fully considered. It can be used to describe the hot carrier cooling; multi-carrier interaction; polaron formation; laser induced phase transition etc. But as an mean field dynamics, it cannot describe trajectory branching and different products in a chemical reaction. The second method is a stochastic method, which uses density matrix to describe the wave function evolution, and natural orbitals to collapse the wave functions upon. It has the decoherence and detailed balance features, as well as the stochastic branching feature. In a way, this is a similar approach to the DISH method $^{22,27,32}$, but with a different and more natural wave function collapsing scheme. Being a stochastic method, one needs to simulate the system multiple times in order to get the statistics. In that sense, it is more expensive than the Boltzmann-TDDFT. In the following, we will first describe these two methods separately. 


\section{BOLTZMANN RT-TDDFT METHOD}

In rt-TDDFT simulation ${ }^{26}$, the equation for the time evolving single particle orbital $\psi_{j}(t)$ is:

$$
i \frac{\partial \psi_{j}(t)}{\partial t}=H(t) \psi_{j}(t)
$$

here $\mathrm{H}(\mathrm{t})$ is the DFT Hamiltonian which depends on the charge density at $\mathrm{t}$, and $\psi_{j}(t)$ has an occupation $o(j)$ which is independent of time. In the simplest case, $o_{j}$ equals 1 for the first $\mathrm{N}$ orbital $\left(N=N_{e} / 2\right.$ for double occupation, and $N=N_{e}$ for single occupation in spin polarized calculations), and zero otherwise, In that case, the whole dynamics is invariant regard to an unitary rotation among $\left\{\psi_{j}(t)\right\}$. Thus in the following, we will drop $o(j)$. We thus should pursue formalism which retain this occupation space rotational invariance.

Our Boltzmann formalism will be based on adiabatic state $\phi_{i}(t)$, which satisfies: $H(t) \phi_{i}(t)=$ $\epsilon_{i}(t) \phi_{i}(t)$. We follow a procedure as in our previous rt-TDDFT work ${ }^{37}$ where we expand $\psi_{j}(t)$ on the basis set of $\phi_{i}(t)$ as:

$$
\psi_{j}(t)=\sum_{i} C(i, j, t) \phi_{i}(t)
$$

We can then convert the Eq.(1) to an equation for the coefficient $C(i, j, t)$

$$
i \frac{\partial C(i, j, t)}{\partial t}=\epsilon_{i}(i, t) C(i, j, t)+\sum_{i^{\prime}} D\left(i, i^{\prime}, t\right) C\left(i^{\prime}, j\right)
$$

Here $D\left(i, i^{\prime}, t\right)=-i<\phi_{i}(t)\left|\frac{\partial}{\partial t}\right| \phi_{i^{\prime}}(t)>$, thus $D(i, i)=0$ and $D\left(i, i^{\prime}\right)=D^{*}\left(i^{\prime}, i\right)$.

Now, in order for our formalism to be occupation subspace rotational invariant, we will concentrate on the density matrix: $d\left(i, i^{\prime}, t\right)=\sum_{j=1}^{N} C(i, j, t) C^{*}\left(i^{\prime}, j, t\right)$. Most importantly, we can define:

$$
T\left(i, i^{\prime}, t\right)=-\sum_{j=1}^{N} 2 \operatorname{Re}\left\{i C^{*}(i, j, t) D\left(i, i^{\prime}, t\right) C\left(i^{\prime}, j, t\right)\right\}
$$

This $T\left(i, i^{\prime}, t\right)$ represents an instantaneous charge flow from i' adiabatic state $\phi_{i^{\prime}}(t)$ to i adiabatic state $\phi_{i}(t)$. Note: $T\left(i, i^{\prime}, t\right)=-T\left(i^{\prime}, i, t\right)$. We first introduce the decoherence effect by making an integration of $T\left(i, i^{\prime}, t\right)$ : 


$$
I\left(i, i^{\prime}, t\right)=\frac{1}{\tau_{i, i^{\prime}}} \int_{0}^{\infty} T\left(i, i^{\prime}, t-t^{\prime}\right) e^{-t^{\prime} / \tau_{i, i^{\prime}}} d t^{\prime}
$$

This $I\left(i, i^{\prime}, t\right)$ will effectively average the charge flow between i and i' over a period of $\tau_{i, i^{\prime}}$ which is the decoherence time between states $\phi_{i}(t)$ and $\phi_{i^{\prime}}(t)$. Longer the decoherence time, longer the average. For example, a long decoherence time will lead to an Fermi golden rule about the transition energy between $\epsilon_{i}-\epsilon_{i^{\prime}}$ which is encoded in the $C$ in Eq.(4) and the phonon frequency which is encoded in the $D\left(i, i^{\prime}, t\right)$ in Eq.(4). If the Fermi golden rule is not satisfied, the $T\left(i, i^{\prime}, t\right)$ will exhibit a sinusoidal oscillation, and the integration of Eq.(5) will yield zero result for $I\left(i, i^{\prime}, t\right)$ (due to coherence cancellation). On the other hand, if the decoherence time is extremely short, then even instantaneous oscillation in $T\left(i, i^{\prime}, t\right)$ will result in real charge transfer.

This integrated quantity $I\left(i, i^{\prime}, t\right)$ is the real charge flow (after considering the coherence interference) which should be subjected to detailed balance. Our basic approach is to modify this charge flow depending on whether such charge flow is from lower adiabatic state to higher adiabatic state, or the opposite. More specifically, we like to introduce the following change to this charge flow in order to enforce the detail balance:

$$
\Delta I\left(i, i^{\prime}, t\right)= \begin{cases}I\left(i, i^{\prime}, t\right)\left(e^{-\left|\epsilon_{i}-\epsilon_{i^{\prime}}\right| / k T}-1\right), & I\left(i, i^{\prime}, t\right)\left(\epsilon_{i}-\epsilon_{i^{\prime}}\right)>0 \\ 0, & I\left(i, i^{\prime}, t\right)\left(\epsilon_{i}-\epsilon_{i^{\prime}}\right) \leq 0\end{cases}
$$

This change will thus reduce the charge flow by a Boltzmann factor if the flow is from an lower energy adiabatic state to a higher energy adiabatic state. Note here, instead of using many-body total energy, we have used single particle eigen energies. In other words, the total energy difference after a single orbital transition is approximated by the single orbital eigen value difference. The charge flow in Eq.(6) is the charge flows between adiabatic states i and i' sum over all time evolving single particle orbital $\psi_{j}(t)$. Thus, in the following, instead of altering each individual single particle wave function $\psi_{j}(t)$, we will alter them as a whole. In this way, we can keep the subspace rotational invariance. We also have $\Delta I\left(i, i^{\prime}, t\right)=-\Delta I\left(i^{\prime}, i, t\right)$ We next add this $\Delta I\left(i, i^{\prime}, t\right)$ to the adiabatic occupation $d(i, i, t)$ as: 


$$
d^{\prime}(i, i, t)=d(i, i, t)+\Delta t \sum_{i^{\prime}} \Delta I\left(i, i^{\prime}, t\right)
$$

The above equation is valid for one integration step of $\Delta t$ time interval. The intention is to use $d^{\prime}$ to replace $d$ in the following step. Alternatively, one can also write the Eq.(7) as:

$$
\frac{\partial}{\partial t} d(i, i, t)=2 \sum_{j=1}^{N} \operatorname{Re}\left(C(i, j, t) \frac{\partial}{\partial t} C^{*}(i, j, t)\right)+\sum_{i^{\prime}} \Delta I\left(i, i^{\prime}, t\right)
$$

with the second term as the Boltzmann correction term. To make a mean field approximation, and keep the formalism remain in the rt-TDDFT representation (e.g., the system is represented by a single SD), we like to modify the coefficient $C(i, j, t)$ in Eq.(2) so we will have the desired $d^{\prime}(i, i, t)$ (after each time integration step $\mathrm{dt}$ ). To do that, we introduce a change $\Delta C(i, j, t)$ to the coefficient $C(i, j, t)$, which satisfies the following linear equation:

$$
\begin{array}{r}
\sum_{i=1}^{M} C\left(i, j_{1}, t\right) \Delta C^{*}\left(i, j_{2}, t\right)+\Delta C\left(i, j_{1}, t\right) C^{*}\left(i, j_{2}, t\right)=0 \\
2 \sum_{j=1}^{N} \operatorname{Re}\left[C(i, j, t) \Delta C^{*}(i, j, t)\right]=\Delta d(i, i, t)
\end{array}
$$

Here $\Delta d(i, i, t)=d^{\prime}(i, i, t)-d(i, i, t)$ as defined in Eq.(7). The first line in Eq.(9) is to satisfy the orthonormal condition for $\psi_{j}(t)$, and the second equation is to yield the modified occupation $d^{\prime}(i, i, t)$ on each adiabatic state $\phi_{i}(t)$. After $\Delta C(i, j, t)$ is solved from Eq.(9) and (10), we can update the wave function after each integration step $\Delta t$ as:

$$
\psi_{j}(t)=\sum_{i}(C(i, j, t)+\Delta C(i, j, t)) \phi_{i}(t)
$$

Note that, if the number $\mathrm{M}$ of adiabatic state $\phi_{i}(t)$ in Eq.(2) is much larger than the number $\mathrm{N}$ of time evolving orbital $\psi_{j}(t)$, Eq.(8) is under determined for $\Delta C(i, j, t)$. Besides, we need to keep in mind that any subspace rotation among $\left\{\phi_{j}(t)\right\}$ will retain the $d(i, i, t)$ and the orhonormal condition. We thus seek the $\Delta C(i, j, t)$ which satisfies the Eq.(9), but also have the minimum amplitudes. We also note that, we have not required any specific values for the off diagonal term of $d\left(i, i^{\prime}, t\right)$. One could impose additional conditions to the change 
of $d\left(i, i^{\prime}, t\right)$ (e.g., proportional to their diagonal values changes in $d(i, i, t)$ and $\left.d\left(i^{\prime}, i^{\prime}, t\right)\right)$ ) to further fix the $\Delta C(i, j, t)$. Right now, we found that fixing $d(i, i, t)$ with minimum change $\Delta C(i, j, t)$ yields good results.

We also note that, in actual integration, we often have $\Delta t$ in the order of $0.1 \mathrm{fs}$ (note, each $\Delta t$ integration involves many smaller dt step integrations of the Eq.(1) on an reduced M dimension basis set of $\left\{\phi_{i}\left(t_{1}\right)\right\}$ with the algorithm described in Ref.37. Due to the finite size of $\Delta t$, the above equations can be modified accordingly, taking into account the finite $\Delta t$ second order effects, for example, to assure that $d^{\prime}(i, i, t)$ remains within the range of $[0,1]$ and the orthonormal condition is satisfied through Eq.(9) (by adding the second order term in $\Delta C(i, j, t))$.

We have used an iterative method to solve Eq.(9). In our test, the solution of Eq.(9) can increase the total computational time by a factor of 2-3 for systems with about $50 \phi_{i}(t)$. The timing however depends on the implementation of the linear solver, as well as the criterion to stop the iteration during the solution of the linear equation.

Lastly, the change in Eq.(11) does change the total energy. To restore the energy conservation, we need to give the energy to the nuclear kinetic energy in the TDF. The $\Delta I\left(i, i^{\prime}, t\right)$ in Eq.(6) is responsible for the total energy change. It can thus be used to define TDF, which is represented by an set of atomic force:

$$
F_{T D F}\left(R_{a}, t\right)=\sum_{i, i^{\prime}} \Delta I\left(i, i^{\prime}, t\right) /\left|d\left(i, i^{\prime}, t\right)\right| R e\left\{<\phi_{i^{\prime}}(t)\left|\frac{\partial H(t)}{\partial R_{a}}\right| \phi_{i}(t)>d\left(i, i^{\prime}, t\right)\right\}
$$

Here $R_{a}$ is the atomic coordinates for atom a, and it is also used here as an atom index. $d\left(i, i^{\prime}, t\right)$ is used to provide the proper phase for the $\phi_{i}(t)$ and $\phi_{i^{\prime}}(t)$ terms. The missed energy can be added back in the direction $\left\{F_{T D F}\left(R_{a}, t\right)\right\}$. More specifically, one can calculate the total energy $E_{D F T}\left(\left\{\psi_{j}(t)\right\}\right)$ after the Eq.(11) update for $\left\{\psi_{j}(t)\right\}$, then calculate the total energy change $\Delta E=E_{t o t}-E_{D F T}-E_{k i n}$, here $E_{k i n}$ is the nuclear kinetic energy, and $E_{t o t}$ is the original conserved total energy. We then rescale the velocity according to:

$$
V^{\prime}\left(R_{a}, t\right)=V\left(R_{a}, t\right)+\alpha F_{T D F}\left(R_{a}, t\right) / M_{a}
$$

here $M_{a}$ is the nuclear mass, the factor $\alpha$ is determined in order to increase the kinetic 
energy by $\Delta E$. Since $\Delta E$ is usually positive (the effect of Boltzmann factor always lowers the total energy, we never found the opposite case), we can always satisfy energy conservation. Actually, there are two solutions, and we should take the small $\alpha$ one (e.g., for small $\Delta t, \alpha$ should also be small, proportional to $\Delta t$ ). This concludes our description for the Boltzmann factor correction for rt-TDDFT simulations. Details testing result will be provided later.

\section{NATURAL ORBITAL BRANCHING}

The above Boltzmann method works well to describe the hot carrier cooling, and how the DFT system jumps from higher energy configurations to lower energy configurations. It is a mean field method for its nuclear movement, so it is deterministic and only has one trajectory. This is good if only the average electronic dynamics is of interest. However, there are cases where the branching of the nuclear movement is of central interest, for example if we like to know what is the probability of different chemical reaction products. In such cases, it is unavoidable to have some stochastic features in our simulation, and multiple runs are needed to get the required statistics. In that sense, the above Boltzmann factor approach is inadequate.

To introduce the stochastic behavior, we will use WFC scheme. In the traditional WFC approach, the many body wave function $\Psi(t)$ is represented as a linear combination of many-body adiabatic states $\Phi_{k}(t)$ as: $\Psi(t)=\sum_{k} C(k, t) \Phi_{k}(t)$. At fixed intervals (e.g., determined by the weighted average decoherence time $\left.{ }^{22}\right)$, the wave function $\Phi_{k}(t)$ will be broken into subset of the adiabatic states with the probability proportional to the amplitudes $|C(k, t)|^{2}$. However, there are many ways to make such collapsing. For example, in the DISH method ${ }^{22}$, one adiabatic state $\Phi_{k}(t)$ is randomly selected according to its amplitude $|C(k, t)|^{2}$. Then with another random number choice, the $\Psi(t)$ is either broken into $\Phi_{k}(t)$,

or into $\sum_{k^{\prime} \neq k} C\left(k^{\prime}, t\right) \Phi_{k^{\prime}}(t)$. There are however issues in such collapsing schemes. First, different pairs $\left(k, k^{\prime}\right)$ of adiabatic states might have different decoherence time $\tau_{k, k^{\prime}}$. The above scheme is difficult to accommodate these individual pair differences. Second, it is hard to justify why the system must collapse into either one adiabatic state, or the sum of the rest adiabatic states. One can imagine, the wave function can collapse into a sum of a subset of adiabatic states. Then, there are many different ways to select the subset. There is even an issue whether the wave function has to collapse into the adiabatic state at all. Based on the 
arguments presented in the introduction section, the wave function should be collapsed into electron wave functions where their nuclear wave functions have become orthogonal to each other. This does not mean such decoherent states must be adiabatic states. For example, in some cases the localized diabatic state might be decoherent between each other more than the adiabatic states. All these mean it will be desirable to have an algorithm which delivers the decoherent states automatically, instead of relying on the many-body adiabatic states in an artificial way. We also like to have a scheme which fit more naturally to the single particle orbital formalism of the DFT calculation, instead of many-body wave functions which do not exist in DFT.

Here we present a natural orbital branching (NOB) method. In this method, for the time dependent Schrodinger's equation, instead of working on the wave functions, we will work on the density matrix $d\left(i, i^{\prime}, t\right)$ on the basis set of single particle adiabatic state $\phi_{i}(t)$. Note in a previous work for nonadiabatic molecular dynamics under $\mathrm{CPA}^{36}$, we have developed a P-matrix formalism based on the density matrix. But that density matrix is an classical assembly of the single particle solution, which is different from our current density matrix, which represents all the electrons in a given system. At the beginning of the simulation, we can have: $d\left(i, i^{\prime}, t=0\right)=\sum_{j=1}^{N} C(i, j, t=0) C^{*}\left(i^{\prime}, j, t=0\right)$ (occupying a set of time evolving wave function $\left\{\psi_{j}\right\}$ ). But as the time evolves, we will no longer have this relationship. The time dependent Schrodinger's equation for the density matrix is:

$$
i \frac{\partial}{\partial t} d\left(i, i^{\prime}, t\right)=\left(\epsilon_{i}(t)-\epsilon_{i^{\prime}}(t)\right) d\left(i, i^{\prime}, t\right)+[D, d]\left(i, i^{\prime}, t\right)
$$

here $[D, d]=D d-d D$ is the anti-commutator of matrix $\mathrm{d}$ and $\mathrm{D}$. So far, this is the same as the wave function based rt-TDDFT. Now, to represent the decoherence between adiabatic single particle orbitals $\phi_{i}(t)$ and $\phi_{i^{\prime}}(t)$, it is common to add an decay term to reduce the off diagonal matrix of $d\left(i, i^{\prime}, t\right)^{38}$. In other words, we can change Eq.(14) to:

$$
i \frac{\partial}{\partial t} d\left(i, i^{\prime}, t\right)=\left(\epsilon_{i}(t)-\epsilon_{i^{\prime}}(t)\right) d\left(i, i^{\prime}, t\right)+[D, d]\left(i, i^{\prime}, t\right)-i d\left(i, i^{\prime}, t\right) / \tau_{i, i^{\prime}}
$$

The last term is only nonzero when $i \neq i^{\prime}$, in other words, $\tau_{i, i}=\infty$. Note that, here, instead of using the decoherence life time $\tau_{k, k^{\prime}}$ between two many-body states $\mathrm{k}$ and $\mathrm{k}$ ', we have used the decoherene life time $\tau_{i, i^{\prime}}$ between two single particle orbital, a description more natural to DFT calculations. In the density matrix formalism, the charge density is 
calculated as : $\rho(r, t)=\sum_{i, i^{\prime}} \phi_{i}(r, t) d\left(i, i^{\prime}, t\right) \phi_{i^{\prime}}^{*}(r, t)$. The kinetic energy is calculated as $E_{k}(t)=-\frac{1}{2} \sum_{i, i^{\prime}}<\phi_{i^{\prime}}\left|\nabla^{2}\right| \phi_{i}>d\left(i, i^{\prime}, t\right)$, and the Hellman-Feynman force can be calculated as: $F_{R}(t)=-\sum_{i, i^{\prime}}<\phi_{i^{\prime}}\left|\frac{\partial H(R)}{\partial R}\right| \phi_{i}>d\left(i, i^{\prime}, t\right)$. Most importantly, as shown in the supplemental information (SI), using this Hellman-Feynman force, Eq.(15) does conserve the total energy, regardless of what $\tau_{i, i^{\prime}}$ are used.

Now, we can diagonalize the density matrix $d\left(i, i^{\prime}, t\right)$, get the eigen vector $C^{\prime}(i, j, t)$ and eigen values $\lambda_{j}(t)$ :

$$
\sum_{i^{\prime}=1}^{M} d\left(i, i^{\prime}, t\right) C^{\prime}\left(i^{\prime}, j, t\right)=\lambda_{j}(t) C^{\prime}(i, j, t)
$$

we then get the natural orbitals:

$$
\theta_{j}(r, t)=\sum_{i=1}^{M} C^{\prime}(i, j, t) \phi_{i}(r, t)
$$

which is the eigen vector of the density matrix. $\theta_{j}(r, t)(\mathrm{j}=1, \mathrm{M})$ are different from the time evolving wave functions $\phi_{j}(r, t)(\mathrm{j}=1, \mathrm{~N})$ in Eq.(1). If there is no decoherence term in Eq.(15), then the first $\mathrm{N} \theta_{j}$ will be the same as the $\phi_{j}(r, t)$ and $\lambda_{j}(t)$ will be the same as 1 (for $\mathrm{j}=1, \mathrm{~N}$ ) and 0 (for other $\mathrm{j}$ ). On the other hand, due to the decoherence term in Eq.(15), we will have $\lambda_{j}(t) \in[0,1]$. We can define an entropy of the set of $\left\{\lambda_{j}(t)\right\}$ as:

$$
S(t)=-\sum_{j}\left[\lambda_{j}(t) \ln \lambda_{j}(t)+\left(1-\lambda_{j}(t)\right) \ln \left(1-\lambda_{j}(t)\right)\right]
$$

Thus, when $\mathrm{S}(\mathrm{t})$ is not zero, the $d\left(i, i^{\prime}, t\right)$ cannot be represented by a single $\mathrm{SD}$. One way to represent $d\left(i, i^{\prime}, t\right)$ is to use an assemble of different decoherent $\mathrm{SD}$, each consisted with $\mathrm{N}$ $\left\{\theta_{j}(t)\right\}$. It is thus natural to set a criterion $S_{c}$, when the entropy $S(t)$ becomes larger/equal to $S_{c}$, we request a WFC event. In this event, we will collapse the wave function into one SD consisted with $\mathrm{N}$ selected $\left\{\theta_{j}(t)\right\}$. Thus, after this WFC event, the entropy $S(t)$ will be reset to zero, and set $\lambda_{j}(t)=1$ for the selected j's and 0 otherwise. Note that, the wave functions (or the density matrix) is not collapsed into a subset of the adiabatic state $\left\{\phi_{i}(t)\right\}$. Instead, it collapsed into a subset of natural orbital $\left\{\theta_{j}(t)\right\}$. The above procedure provides a way to deal with the different decoherence time $\tau_{i, i^{\prime}}$ for different adiabatic pair $\left(i, i^{\prime}\right)$. Thus, in our scheme, although the decoherence happens between different single particle adiabatic orbital pairs, the WFC happens on the basis of natural orbitals. This is a major difference 
between out NOB scheme and the DISH scheme ${ }^{22}$. Another difference is our Eq.(15). If the original Eq.(14) is used as in DISH, then in our scheme, there will be no WFC. The use of $S_{c}$ provides a natural choice to decide when to do WFC. It is not based on an regular time interval determined by $\tau_{i, i^{\prime}}$. Basing directly on $\tau_{i, i^{\prime}}$ (or their average) can cause some problems. For example, if the off diagonal $D\left(i, i^{\prime}, t\right)$ in Eq.(3) is small, at time equals $\tau_{i, i^{\prime}}$, there might not be large adiabatic state mixing, it is thus not necessary to break up the wave function $\psi_{j}(t)$ (since $\psi_{j}(t)$ might still be the same as the adiabatic state $\phi_{i}(t)$ ). As we will find out later, in our scheme, the interval of wave function collapsing varies a lot depending on the dynamic situation in different time period.

Now, lets discuss the procedure to select $\mathrm{N}$ orbital $\mathrm{j}$ from $\mathrm{M}$ possible candidates based on probability $\left\{\lambda_{j}(t)\right\}$. We require that the probability for each $\theta_{j}(t)$ to be selected is proportional to $\left\{\lambda_{j}(t)\right\}$, this is important so an assemble average after the collapsing events will retain the occupation of each $\theta_{j}(t)$. This is a sampling without replacement problem with desired unequal probability of selection ${ }^{39}$. We follow the procedure by Sampford ${ }^{39}$ with a small modification. In Sampford's procedure, sampling with replacement (which means the selected $\mathrm{j}$ will not be removed from the $\mathrm{M}$ candidate pool) is used. The first draw (selecting one $\mathrm{j}$ from the $\mathrm{M}$ candidates) is done according to their probabilities $\lambda_{j}(t) / N$. The subsequent N-1 draws are done with their probabilities proportional to $\lambda_{j}(t) /\left(1-\lambda_{j}(t)\right)$. However, if any of the $\mathrm{j}$ have been draw more than once in the above $\mathrm{N}$ draws, the whole draw will be abandon, and the draw will restart from beginning. This procedure is simple. However, in our case, we have many $\mathrm{j}$ with their $\lambda_{j}(t)$ close to 1 , this will lead to extremely high probability for one of the j's to be draw more than once. To avoid this, we have made the following modifications. First, we have select a small $\delta$ (e.g., 0.01). Let's assume there are $\mathrm{Q}$ j's with $\lambda_{j}(t)>1-\delta$. For these $\mathrm{Q} \mathrm{j}$ 's, for each $\mathrm{j}$, we randomly determine whether it will be selected according to its probability $\lambda_{j}(t)$. Let's assume q of these j's have been selected in this way (usually $\mathrm{q}$ is rather close to $\mathrm{Q}$, since these $\lambda_{j}(t)$ are all close to 1 ). Now, we place the rest M-Q j's in the Sampford's method, and require it to select N-q j's proportional to their probability $\lambda_{j}(t)$. If there is double drawing in Sampford's procedure, we repeat it until it is successful. Since we have removed the j's with close to one $\lambda_{j}(t)$, the probability for double drawing has been significantly reduced, e.g., the successful rate can be about $20 \%$. Fig. 1 show a typical case with $\mathrm{S}=1.7$, and $\mathrm{N}=12 \theta_{j}(t)$ is selected from $\mathrm{M}=25$ possible states. The $\lambda_{j}(t)$ (and $\left.1-\lambda_{j}(t)\right)$ are shown in logarithmic scale, together with the 
actual probability $p_{j}$ tested with a million successful selections with $\delta=0.01$. We can see $p_{j}$ is very close to $\lambda_{j}(t)$. Note, the above selection is the same as to select all possible $C_{M}^{N}$ occupation configurations $S(u)$ each with a probability:

$$
P(S(u))=K(N, M) \prod_{u=1}^{N} \frac{\lambda_{j(u)}(t)}{\left(1-\lambda_{j(u)}(t)\right)}\left(N-\sum_{u=1}^{N} \lambda_{j(u)}(t)\right)
$$

where $\mathrm{K}(\mathrm{N}, \mathrm{M})$ is an overall normalization factor depends only on $\mathrm{N}$ and $\mathrm{M}$, and $j(u)$ is the selected j index of $S(u)$. It is interesting to note that $P(S(u))$ does not simply proportional to $\prod_{u=1}^{N} \lambda_{j(u)}(t)$.

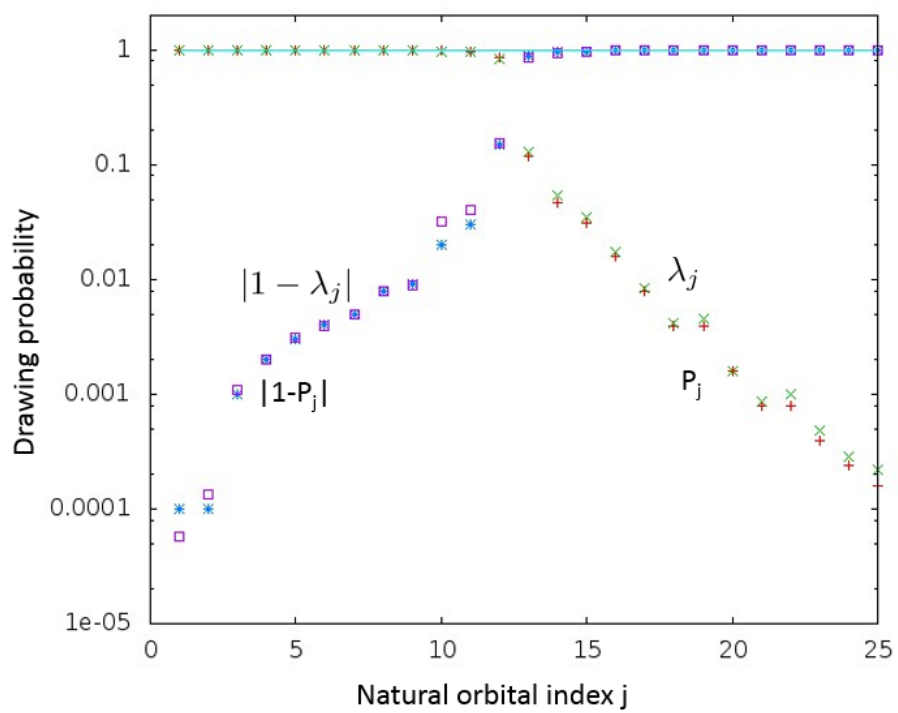

FIG. 1. The selected probability $p_{j}$ compared with the $\lambda_{j}$ using a modified Sampford algorithm with $\delta=0.01$. The entropy of the $\left\{\lambda_{j}\right\}$ set is 1.75

After the N j's subset $S(u)$ is selected, we can set $\lambda_{j^{\prime}}(t)=1$ for all the selected $j^{\prime}=j(u)$ $(\mathrm{u}=1, \mathrm{~N})$, and $\lambda_{j^{\prime \prime}}=0$ for the unselected j". We thus reset the $S(t)$ to zero. However, this should not be the final decision for whether this selection is successful. First note, between two consecutive WFC events, the total energy is conserved by Eq.(15). Like in the other HS or WFC schemes, it is critical to make sure the hopping/collapsing event can satisfy the total energy conservation using the transition degree of freedom (TDF), so the detailed balance will be restored. To do this, one first evaluates the DFT energy $E_{D F T, a f t e r \_W F C}$ using the selected $\left\{\theta_{j^{\prime}}(t)\right\}$ as the occupied wave functions. In principle, this DFT energy evaluation should be straight forward, no SCF iteration should be needed. However, in practice, there is a subtle issue in our adiabatic state based calculation using Eq.(2) and Eq.(17) ${ }^{37}$. Before 
the collapse, the $\left\{\theta_{j^{\prime}}(t)\right\}$ can all be represented by the adiabatic state $\phi_{i}(t)$ according to Eq.(17). However, after the collapse, the charge density is changed to $\rho^{\prime}(r, t)$, thus $\phi_{i}(t)$ will also be changed to $\phi_{i}^{\prime}(t)$. If the number $\mathrm{M}$ of the adiabatic basis set is not very large (hence $\left\{\phi_{i}^{\prime}(t)\right\}$ is not a complete basis set), the $\left\{\theta_{j^{\prime}}(t)\right\}$ can no longer be represented by $\left\{\phi_{i}^{\prime}(t)\right\}$. Usually this is a small error due to the finite number of M. However, without fixing, it can cause numerical instability. We have developed a procedure to solve this problem (as described in the SI), where the $\phi_{i}^{\prime}(t)$ is solved iteratively, while the calculation of charge density is based on $\left\{\theta_{j^{\prime}}^{\prime}(t)\right\}$ which is a projection of $\left\{\theta_{j^{\prime}}(t)\right\}$ to the $\left\{\phi_{i}^{\prime}(t)\right\}$ basis set. Such a selfconsistent solution guarantees that $\left\{\theta_{j^{\prime}}^{\prime}(t)\right\}$ can be represented by the adiabatic states $\left\{\phi_{i}^{\prime}(t)\right\}$ of charge density $\rho^{\prime}(t)=\sum_{j^{\prime}}\left|\theta_{j^{\prime}}^{\prime}(t)\right|^{2}$. Thus after the WFC, the system will restart with a set of occupied wave functions: $\left\{\theta_{j^{\prime}}^{\prime}(t)\right\}$.

After the $E_{D F T, \text { after_WFC }}$ is calculated, if $\Delta E=E_{D F T, \text { after_WFC }}-E_{D F T, \text { before_WFC }}>0$ we need to have a TDF to determine whether the increased potential energy can be provided by the kinetic energy in the TDF; while if $d E \leq 0$, we need to give the extra kinetic energy to the TDF. Our wave function collapsing can be considered as a transition between all the unselected natural orbitals $\left\{\theta_{j^{\prime \prime}}\right\}$ to all the selected natural orbitals $\left\{\theta_{j^{\prime}}\right\}$. We thus can calculate the TDF force direction as:

$$
F_{T D F}\left(R_{a}, t\right)=\sum_{j^{\prime} \in N ; j^{\prime \prime} \notin N}\left(1-\lambda_{j^{\prime}}\right) \lambda_{j^{\prime \prime}} R e\left\{<e^{i \beta_{j^{\prime}}} \theta_{j^{\prime}}\left|\frac{\partial H(t)}{\partial R_{a}}\right| e^{i \beta_{j^{\prime \prime}}} \theta_{j^{\prime \prime}}>\right\}
$$

Here we have added a random phase factor $e^{i \beta_{j^{\prime}}}$ to each natural orbital $\theta_{j^{\prime}}$. One can consider this as an additional random factor in the stochastic process. Given this $F_{T D F}$ and $\mathrm{dE}$, we can judge whether the needed energy (in case $\Delta E>0$ ) can be provided from the nuclear kinetic energy using Eq.(13). If that can be provided by the kinetic energy, or if $\Delta E<0$, we can adjust the nuclear velocity by Eq.(13). If on the other hand, $\Delta E>0$ cannot be provided by the nuclear kinetic energy as described by Eq.(13), then we will repeat the $\mathrm{N}$ j's selection process, until one selection where Eq.(13) can satisfy the $\Delta E$. In practice, we found that, typically 1 to 2 selection will be enough to satisfy the energy conservation. Only occasionally, more than 2 selections are needed. Our strategy is that, when $S(t)=S_{c}$, we keep making selection until the energy conservation is successful. So, at each $S(t)=S_{c}$ point, we will always have a WFC event. After this event, and after the rescaling of the velocity, our simulation can continue with $\left\{\theta_{j^{\prime}}^{\prime}(t)\right\}$ as the initial states. This concludes our 
NOB procedure description.

\section{RESULTS AND DISCUSSIONS}

To test our methods, we choose to study radiolysis problem for molecules. In the radiolysis ${ }^{40}$,the molecule is first ionized (e.g., losing one electron into a +1 state) by either electron beam in electro microscope or by a ultroviolet light. Subsequently, the molecule can break down into fragments. This is an important problem for material decay, but there are insufficient theoretical studies due to the lack of appropriate ab initio methods ${ }^{41}$. This is also a complicated problem which can involve different physical phenomena like Auger cascade, Coulomb explosion, hot carrier cooling and electron-phonon interaction. To test our methods, we will focus on the electron-phonon interaction and hot carrier cooling, while ignoring other possible phenomena at this moment. We have chosen molecule $\mathrm{C} 3 \mathrm{H} 7 \mathrm{OH}$ to carry out our test. Plane wave pseudopotentials are used to represent the Hamiltonian, with a 50 Ryd cut off for the plane wave kinetic energy, and SG15 optimized norm-conserving Vanderbilt pseudopotential (ONCVPSP) is used. General gradient approximation (GGA) exchange-correlation functional is employed. The calculation is done with spin polarization. The molecule is placed in a $8 \times 8 \times 8 \AA$ p periodic box. At neutral charge, the system has 26 electrons. An ab initio molecular dynamics (AIMD) has been performed at room tem-

perature with neutral charge. After the equilibrium has been reached, we carry out the rt-TDDFT simulation.

First, the Kohn-Sham orbital eigen energies, and some of their wave functions squares are shown in Fig.2 at neutral charge. We can see that the first valence state is about $18 \mathrm{eV}$ below the highest occupied molecular orbital (HOMO). The wave functions have different characters, localized at different bonds. We like to mimic the situation where one of the electron has been ionized (e.g., by an high energy electron beam, or ultraviolet light). In reality, this means the system is transited into one of the higher energy many-body excited states, while its nuclei remain at $\mathrm{t}=0$ positions (under the instantaneous transition approximation). We thus have one hole in one of the valence orbital in one spin, and we carry out a constraint occupation spin polarized SCF DFT calculation. Such constraint DFT result will approximate the corresponding many-body eigen state at a given occupation configuration. This is the $\mathrm{t}=0$ electronic structure configuration for our rt-TDDFT simulation. 


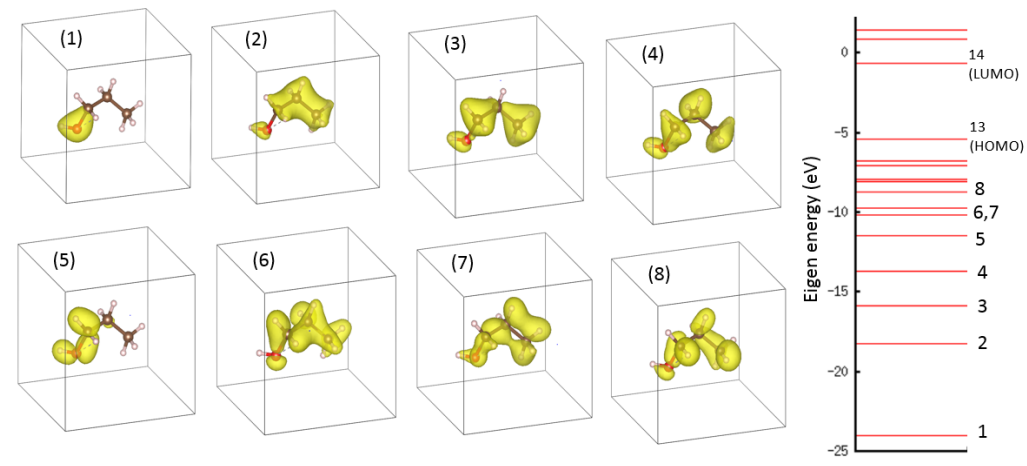

FIG. 2. The DFT eigen energy spectrum (right panel) and the first 8 valence state wave function squires for the neutral charge $\mathrm{C} 3 \mathrm{H} 7 \mathrm{OH}$ molecule.

Our rt-TDDFT simulation will be based on the adiabatic state representation formalism as described in Ref.37. This allows us to use a relative large $\Delta t$ (0.1 fs). Note, our subsequent Boltzmann and NOB operations will all be based on this $\Delta t$ interval, although within each $\Delta t$ step, there are many smaller time evolution steps on a reduced M dimension Hamiltonian, here $\mathrm{M}$ is the number of adiabatic states used in Eq.(2). For details of this integration scheme, we refer to Ref.37. We first carried out a plain rt-TDDFT by removing one electron from valence state 4 in one spin (hence the system will be +1 charged), roughly at the middle of the valence bands as shown in Fig.2. Such rt-TDDFT simulation can be used to study phenomena like ultra-fast dynamics and molecule dissociation before hot carrier cooling becomes important. In recent years, there are many of such rt-TDDFT simulations to study different phenomena ${ }^{42-44}$. Fig.3(a) shows the potential energy (DFT energy, excluding the nuclear kinetic energy, the same terminology below) as a function of integration time. We see that, although there are large energy changes and oscillations, there is no clear trend of the DFT total energy reduction (hot carrier cooling). This is because the plain rt-TDDFT does not have the detailed balance. We have plotted the adiabatic eigen energies $\epsilon_{i}(t)$ as functions of time t in Fig.3(b). As we can see, there are large oscillations. Nevertheless, the initial structure in the energy spectrum remain, and there is still a large band gap between the lowest occupied molecule orbital (LUMO) and highest occupied molecule orbital (HOMO). We have also plotted the occupations of the 13 adiabatic valence states calculated as: $o_{i}(t)=\sum_{j=1}^{N}|C(i, j)|^{2}$ in Fig.3(c). The state 4 has a zero occupation at $\mathrm{t}=0$, but gains its occupation after 20 fs. At 100 fs, there are still many states having relatively large amplitudes especially among valence states 6 to 13 . Thus there is no clear picture of hot 
carrier cooling. Instead the holes are roughly equally distributed among the valence bands.
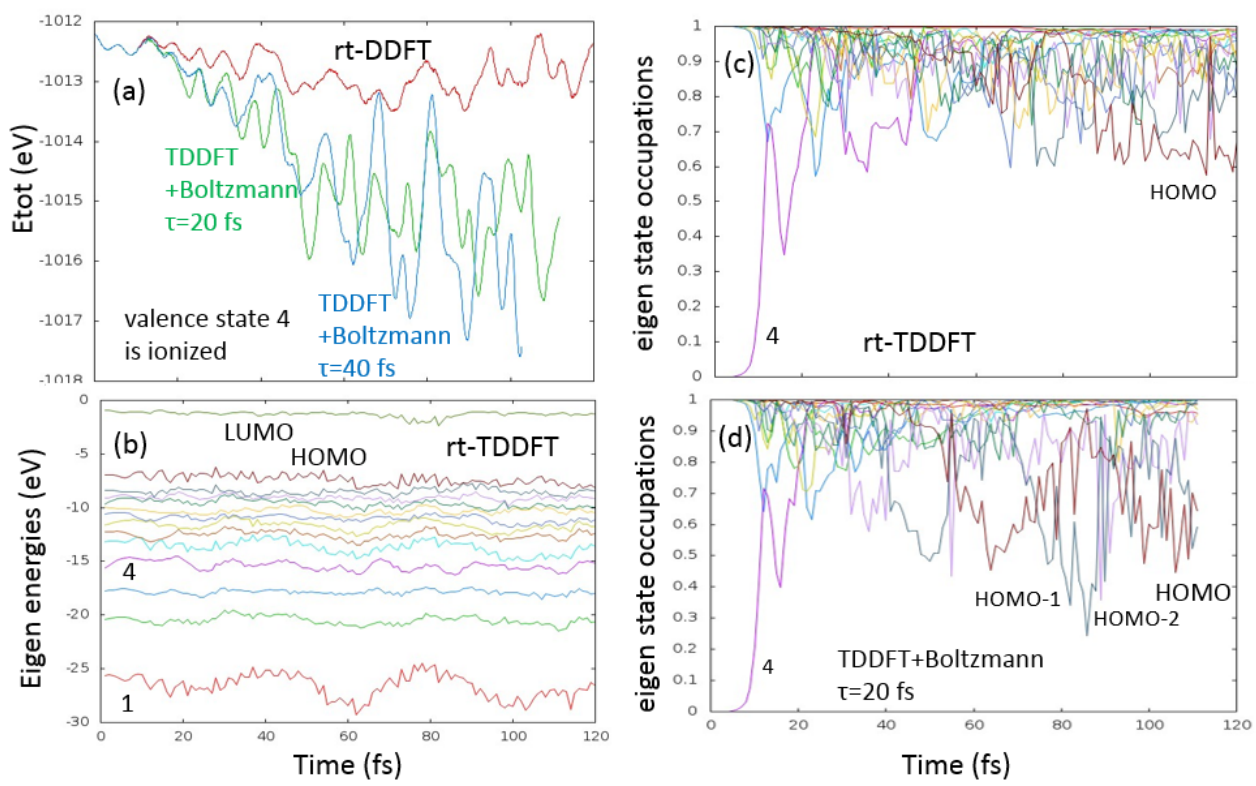

FIG. 3. (a) The potential energy changes during a rt-TDDFT simulation when valence state 4 is ionized at $\mathrm{t}=0$. Three cases are tested: (1) the pure rt-TDDFT simulation without Boltzmann factor; (2) the rt-TDDFT simulation with Boltzmann factor and decoherence time $\tau$ equals 20 fs; (3) the rt-TDDFT simulation with Boltzmann factor and decoherence time $\tau$ equals $40 \mathrm{fs}$; (b) The adiabatic state eigen energy changes as functions of time during rt-TDDFT simulation. The ones with Boltzmann factor (not shown) are qualitatively similar; (c) The occupation changes on the adiabatic states during a pure rt-TDDFT simulation. At the beginning, the state 4th is empty; (d) The occupation changes on the adiabatic states for the rt-TDDFT with Boltzmann factor with $\tau$ equals 20 fs.

We then carry out Boltzmann rt-TDDFT calculation for the above system. There are different ways to calculate $\tau_{i, i^{\prime}}$ for each individual pairs (i,i'), e.g., from their energy difference fluctuations from Fig.3(b) ${ }^{36}$. However, here we will first use an uniformed $\tau_{i, i^{\prime}}=20$ fs to represent this effect as we found $\tau$ is usually around this range for many different systems ${ }^{36,45}$. The potential energy as a function of time is shown in Fig.3(a) in comparison with the plain rt-TDDFT result. As we can see, the potential energy in Boltzmann factor simulation decays significantly, representing hot carrier cooling. The individual eigen state energies are qualitatively similar to the plain rt-TDDFT results shown in Fig.3(b). However, the adiabatic state occupations looks rather different, as shown in Fig.3(d). In particular, at $100 \mathrm{fs}$, the hole occupations are concentrated on the few near HOMO states, much more narrowly distributed than the pure rt-TDDFT case as shown in Fig.3(c). To test the effect of $\tau_{i, i^{\prime}}$, we have also done a Boltzmann simulation with $\tau=40 \mathrm{fs}$. The potential energy 
result is also shown in Fig.3(a). As we can see, its results are rather similar to that of the $\tau=20$ fs result in their overall trends. Thus, within a reasonable range of $\tau$, the result is insensitive to the exact $\tau$ value (on the other hand, if $\tau$ is $\infty$, the Boltzmann method becomes the pure rt-TDDFT method). Overall, we see the effects of detailed balance for the hot carrier cooling in the Boltzmann rt-TDDFT simulations.

Although the Boltzmann rt-TDDFT has a detailed balance, its nuclear trajectory follows a mean field path. There is no stochastic effects, thus cannot be used to describe different possible reaction paths and products. In our case, under the high energy ionization (photolysis), the molecule can be broken into many fragments. We found that there is no molecule breaking when the 4 th valence state is ionized in both of our plain rt-TDDFT simulation and Boltzmann rt-TDDFT simulation. However, if we ionize the first valence band state, in both rt-TDDFT and Boltzmann rt-TDDFT simulation, we found the OH group will fly away from the rest of the molecule. This is a pure dynamic process due to the initial hole concentrate at the O-C bond as shown in Fig.2. Thus, this happens to both rt-TDDFT and Boltzmann rt-TDDFT. But there is no other fragmentation happens. We thus carried out 23 NOB simulations (with different random number seeds) for the valence state 1 ionization. Although the initial condition is the same, we get many different fragments due to the stochastic feature of the simulation, as summarized in Table.I (for the different fragments and their counts of appearance in the 23 runs at the end of $200 \mathrm{fs}$ ). As we can see, the NOB, has a higher tendency to break the molecule into more fragments. Perhaps this is because Boltzmann rt-TDDFT is a mean field method for the nuclear trajectory, the method cannot break the symmetry and select one particular fragment breaking path instead of another. As a result, it has a smaller tendency to break up the molecule.

The potential energies as functions of time for the 23 different simulations when the first valence state is ionized are shown in Fig.4(a). As we can see, there is a wide range of trajectories. However, the trajectories cluster into different groups, especially for the initial major WFC. One happens around 50 fs, another happens around 100 fs. Note, in all these trajectories, the $\mathrm{OH}$ group will separate from the molecule in the first $10 \mathrm{fs}$, as a dynamic process, same as in the plain rt-TDDFT and Boltzmann rt-TDDFT simulations. After the $\mathrm{OH}$ flies away, in some cases, they will suddenly dissociate, but in other cases, they stick together. For the remaining $\mathrm{CH} 2 \mathrm{CH} 2 \mathrm{CH} 3$, in most cases, they will become $\mathrm{CH} 3 \mathrm{CHCH} 3$ fragment. But in some cases, the $\mathrm{H}$ can fly away, thus generate some other 3 carbon 
fragments. The $\mathrm{H}$ dissociation process can be a result of the secondary collision by the flyaway $\mathrm{H}$ (from the initial $\mathrm{OH}$ ), but can also be due to the thermal vibration of the 3 carbon fragment itself without collision. Surprisingly, the C-C bond was never broken by itself in this valence state-1 ionization case, despite the large ionization energy. A detail analysis of the trajectories shows that, all the 2 carbon and 1 carbon products in Table.I are the results of secondary collision by the fly-away $\mathrm{OH}$, or dissociated $\mathrm{O}$, or $\mathrm{H}$, to the periodic neighbours of the molecule. So, if we were simulating an isolated molecule, they should not exist. The same is true for $\mathrm{CH} 3 \mathrm{CO}, \mathrm{CHO}$, and $\mathrm{H} 2 \mathrm{O}$ fragments, they are all the results of secondary collision.

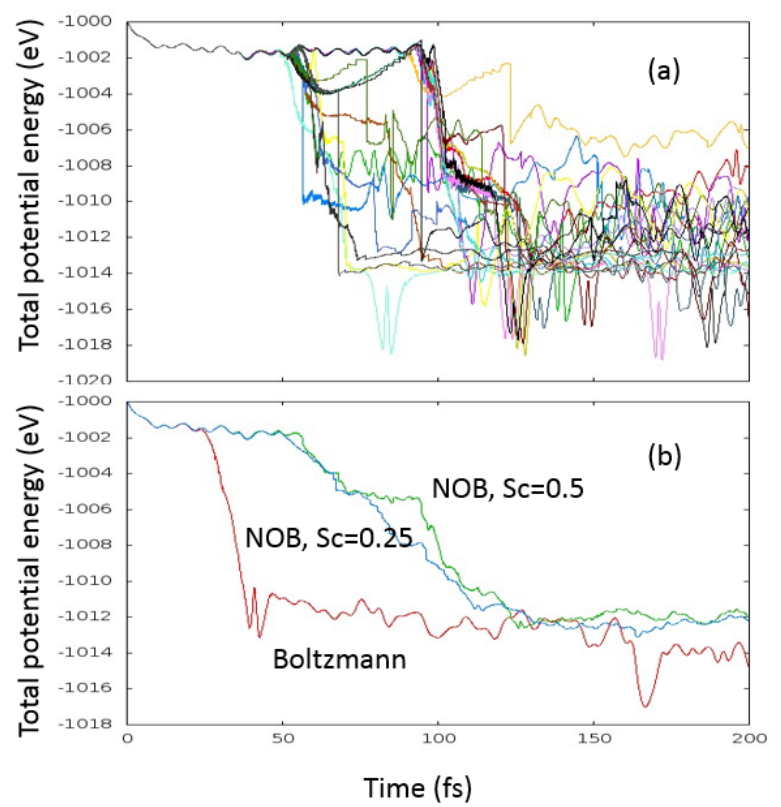

FIG. 4. (a) The individual trajectory potential energies when the valence state 1 is ionized using NOB algorithm; (b) The average energy for the 23 trajectories in (a), for $\mathrm{Sc}=0.5$ and $\mathrm{Sc}=0.25$, in comparison with Boltzmann rt-TDDFT simulation potential energy. All the simulations have used $\tau_{i, i^{\prime}}=20 f s$.

In the above tests, we have used $S c=0.5$. In order to test the effect of $S c$, we have repeated the 23 trajectory calculations with $S c=0.25$. The individual trajectories are very different. However, the average potential energy of these 23 trajectories (also shown in Fig.4(b)) are rather similar to the $S c=0.5$ result. This indicates that the final assembly result is insensitive to the exact value of $S c$ used. In Fig.4(b), we also show the Boltzmann rt-TDDFT result for valence state-1 ionization. We see that the Boltzmann result decay faster than the NOB result at the beginning. Also, its final potential is slightly lower. This is 
TABLE I. The final fragments and their the number of their appearance in the 23 NOB simulations after $200 \mathrm{fs}$ simulations. Some of the final fragments are results of collisions with periodic neighboring molecules.

\begin{tabular}{cc|cc|cc}
\hline \hline Fragment & $\mathrm{N}$ & Fragment & $\mathrm{N}$ & Fragment & $\mathrm{N}$ \\
\hline $\mathrm{CH} 3 \mathrm{CH} 1 \mathrm{CH} 3$ & 10 & $\mathrm{CHCH}$ & 1 & $\mathrm{OH}$ & 6 \\
$\mathrm{CH} 3 \mathrm{CHCH} 2$ & 3 & $\mathrm{CH} 3 \mathrm{CO}$ & 1 & $\mathrm{H} 2$ & 3 \\
$\mathrm{CH} 3 \mathrm{CHCH}$ & 3 & $\mathrm{CHO}$ & 4 & $\mathrm{O}$ & 12 \\
$\mathrm{CH} 3 \mathrm{CCH} 3$ & 2 & $\mathrm{CH} 3$ & 3 & $\mathrm{H}$ & 30 \\
$\mathrm{CH} 3 \mathrm{CH}$ & 1 & $\mathrm{CH} 2$ & 3 & & \\
$\mathrm{CH} 2 \mathrm{CH} 2$ & 1 & $\mathrm{H} 2 \mathrm{O}$ & 1 & & \\
\hline \hline
\end{tabular}

because the Boltzmann result does not have any other molecule breaking besides the initial $\mathrm{OH}$ one, while the NOB can generate more molecule fragments, which cost energy, thus makes their potential energies higher (and kinetic energy lower).

To illustrate the dynamics processes in more details, we have shown in Fig.5(a) the increase of $S(t)$ and the wave function collapsing events for one particular NOB trajectory (call it trajectory-1) using $S c=0.5$. We see that this trajectory has a slow $S(t)$ increase at the beginning. This is because at the first, the hole is concentrate on state 1 and 2 as shown in Fig.5(d), and only these two states have energy crossing with each other, as shown in Fig.5(c). However, after 90 fs, several big WFC events happen, and there is a period with frequent WFC, until the system potential energy is completely relaxed to the equilibrium one (Fig.5(b)). This fast WFC period also corresponds to the jump of the hole concentration to the higher energy states as shown in Fig.5(d). Since these higher energy adiabatic eigen energies are close to each other as shown in Fig.5(c), they have much faster state mixing, which causes $S(t)$ to increase rapidly, and leads to more frequent WFC until the system reach equilibrium, with the hole resides in the HOMO level.

To demonstrate the NOB algorithm can lead to different fragments, we show in Fig.6 four different trajectories. We have calculated the bond lengths at $t=0$ and their subsequent changes. They are numerated in the inset of Fig.6(a). The trajectory in Fig.6(a) is the trajectory-1 shown in Fig.5 when the first valence state is ionized. As we can see, the O-C bond is dissociated immediately after the initial ionization because the strong localization of the valence state 1 shown in Fig.2. At about $100 \mathrm{fs}$, in trajectory-1, the O-H bond of the fly-away $\mathrm{OH}$ group is dissociated. This happens when $\mathrm{OH}$ group is isolated, perhaps due to its electronic structure WFC, and it follows the rapid WFC period shown in Fig.5(a). On the 

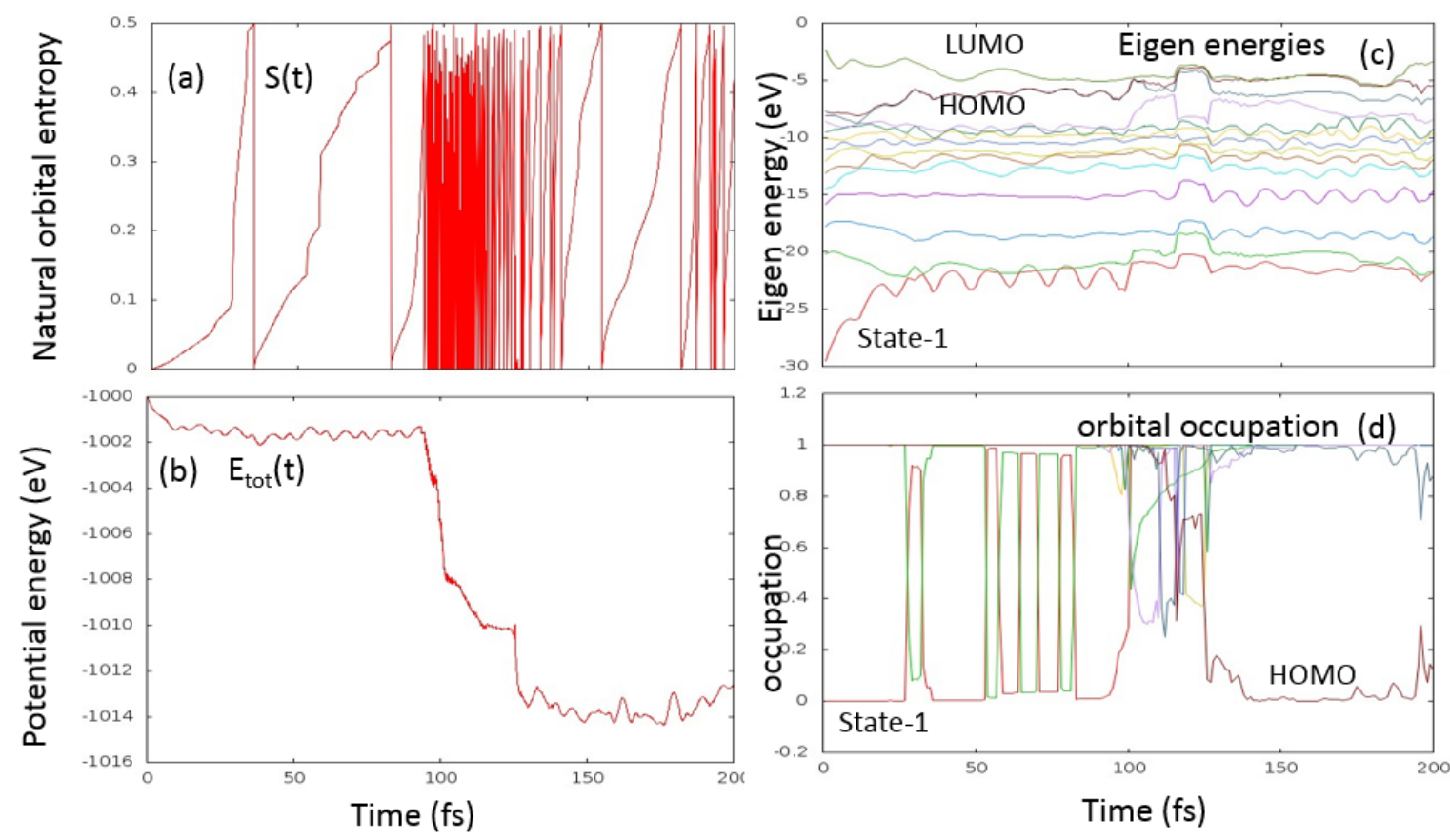

FIG. 5. The details of one particular trajectory (trajectory-1) in Fig.4(a) when valence state 1 is ionized at t $=0$ using $S c=0.5$. (a) The increase of $S(t)$ as a function of time; (b) The potential energy of this trajectory as a function of time; (c) The spin=1 single particle adiabatic eigen state energies $\epsilon_{i}(t)$. Note, at $\mathrm{t}=0$, the first state is empty for $\operatorname{spin}=1 ;(\mathrm{d})$ The adiabatic state occupation $o_{i}(t)=d(i, i, t)$. Note, the sudden change of occupation between state 1 and 2 before $80 \mathrm{fs}$ is mostly caused by the eigen energy crossing between these two states, which leads to a change of definition for which state is 1 , which is 2 . The occupations for different eigen states are color coded in the same way as in (c).

other hand, for trajectory-2 shown in Fig.6(b), the OH group is never dissociated. Instead, two $\mathrm{H}$ atoms dissociated from the carbon 3 group, perhaps due to thermal vibrations with the heat generated from the relaxation of the initial high energy ionization (the molecule can reach 4000-8000 $\mathrm{K}$ in temperature due to the energy gained from the hot hole cooling). In Fig.6(c), We show a trajectory when the valence state 7 is ionized. In this case, none of the bond is broken, the molecule remains as a whole. On the other hand, in trajectory-2 shown in Fig.6(d), one C-C bond is broken, the final fragments are $\mathrm{CH} 2 \mathrm{OH}$ and $\mathrm{CH} 2 \mathrm{CH} 3$. We thus see that, it is not just the ionization energy, but also the ionized orbital, which determine what bonds will be broken. The stochastic feature in NOB allows the description of different reaction paths from the same initial condition.

Overall, we see that our NOB is able to calculate the molecular breakdown in the radiolysis process. It can provide the rates for different molecule fragment products. It is worth 

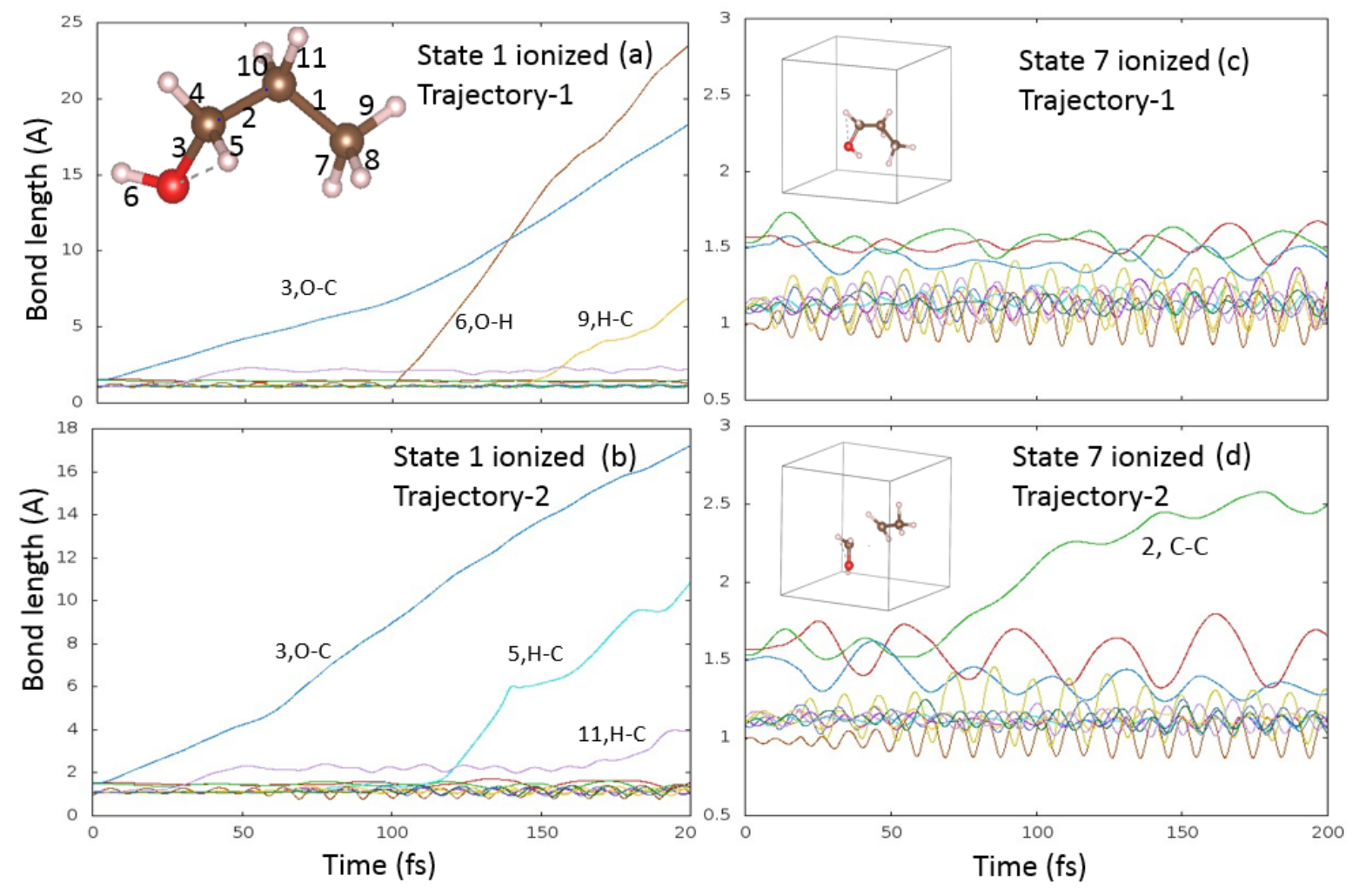

FIG. 6. The bond breaking in different NOB trajectories at different initial valence state ionizations. (a) The trajectory- 1 when valence state 1 is ionized. The number in the bond (e.g, 3,O-C) corresponds to the numbers in the inset; (b) The trajectory-2 when valence state 1 is ionized; (c) The trajectory-1 when valence state 7 is ionized. See Fig. 2 for the initial valence state wave functions and eigen energy; (d) The trajectory-2 when valence state 7 is ionized.

to mention that one does not need to carefully analyse the process and provide the specific many-body state configurations $\mathrm{k}$ before the simulation. Such analysis is possible for small molecules, but becomes increasingly complicated for large systems, and for high energy excitation cascade processes like we tested here. In our calculation, all the selections are done automatically, and there is no need to numerate all the possible many-body excited states $\mathrm{k}$, which can be extremely large. Such automatic selection of the states to be collapsed on (the natural orbital) can be beneficial for high throughput simulations for large systems.

In terms of computational time, the NOB is almost the same as the original rt-TDDFT. The density evolution of Eq.(15) has a similar cost as our original adiabatic basis set integration scheme ${ }^{37}$. The main cost is to the calculation of the adiabatic state $\phi_{i}(t)$. The off diagonal decay term in Eq.(15) does not affect the iterative convergence of the $\phi_{i}(t)$. One can also use other exchange-correlation functional in the simulation, e.g, the hybrid 
exchange-correlation functional, without changing the algorithm itself. The natural orbital selection does not cost extra time. In most case, only one extra SCF DFT calculation is needed to evaluate the total energy after each WFC, and to determine whether a WFC can be accepted using Eq.(13). Since the WFC happens only in about 5\% (when $S c=0.5$ ) to $10 \%$ (when $S c=0.25$ ) of the total MD steps (when a $\Delta t=0.1$ fs is used), this only constitutes about 5\% to $10 \%$ cost increase. Both NOB and Boltzmann method make the rt-TDDFT simulation more stable when using the adiabatic basis set integration scheme ${ }^{37}$. This is because there are less occupation in the higher energy adiabatic state basis functions near the truncation of the basis set, which makes the algorithm more stable. For the plain rt-TDDFT, the algorithm might diverge after a long time evolution (when the higher adiabatic basis become populated), a consequence of the lack of detailed balance. In both NOB and Boltzmann method, the simulation can continue without the divergence problem. For our test system, for a 2000 step (200 fs) simulation, it takes about 2 hours on a 4 GPU workstation.

\section{CONCLUSION}

In summary, we have introduced two new schemes for nonadiabatic molecular dynamics under rt-TDDFT formalism. One is the Boltzmann factor scheme which uses the decoherence time and restores the detailed balance in the simulation. It thus can be used to describe the hot carrier cooling with electron-phonon interaction and backreaction from electron dynamics to nuclear dynamics. However, in terms of nuclear dynamics, it uses a mean field approach, much like the original rt-TDDFT. It thus cannot be used to describe the nuclear trajectory branching, and different paths and products of an chemical reaction. The second method is a natural orbital branching (NOB) scheme based on the stochastic WFC approach. This is close to the DISH ${ }^{22}$ method, but with critical difference in the collapsed wave function selection and the time evolution of the wave function. We use a density matrix

formalism for time propagation, and natural orbital basis to collapse the wave function upon. Thus, unlike most many-body based methods, here we do not collapse the wave function into an many-body adiabatic state, or a SD consisted with single particle adiabatic states. Instead, we collapse the wave function into a set of natural orbital. The selection process is simple, and do not need to deal with the exponentially increasing (with system size) number 
of possible adiabatic state SDs. We feel this is a more natural way to collapse the wave function to. We have tested our methods on the molecule radiolysis problem. We found they can efficiently study such problems delivering what the algorithms are designed to do. For the Boltzmann method, the computational time increases by a factor or 2-3 due to the solution of a linear equation, although this could be improved by further code optimizations. For the NOB method, the computational time is about the same as the plain rt-TDDFT method. These two methods make the rt-TDDFT integration more stable than the original plain TDDFT calculation. We expect them to find wide range of applications in different nonadiabatic problems.

\section{ACKNOWLEDGMENTS}

This work is supported by the Director, Office of Science, the Office of Basic Energy Sciences (BES), Materials Science and Engineering (MSE) Division of the U.S. Department of Energy (DOE) through the theory of material (KC2301) program under Contract No. DEAC02-05CH11231.

1 J. C. Tully, "Perspective: Nonadiabatic Dynamics Theory". J. Chem. Phys. 137, 22A301 (2012).

2 D.R. Yarkony, "Nonadiabatic Quantum Chemistry: Past, Present, and Future". Chem. Rev. 112, 481-498 (2012).

3 M. Born, K. Huang, "Dynamics Theory of Crystal Lattices", New York, Oxford University Press (1954).

4 M.H. Beck, A. Jackle, G.A. Worth, H.D. Meyer, "The Multiconfiguration Time-Dependent Hartree (MCTDH) Method: A Highly Efficient Algorithm for Propagating Wavepackets". Phys. Rep. 324, 1-105 (2000).

5 B.F.E. Curchod, U. Rothlisberger, I. Tavernelli, "Trajectory-based nonadiabatic dynamics with time-depedent density functional theory", ChemPhysChem 14, 1314 (2013).

6 E.J. Heller, "The Semi-Classical Way to Molecular-Spectroscopy", Acc. Chem. Res. 14, 368 (1981). 
7 B.F.E. Curchod, T.J. Martinez, "Ab initio Nonadiabatic Quantum Molecular DYnamics", Chem. Rev. 118, 3305 (2018).

8 J. C. Tully, "Molecular dynamics with electronic transitions", J. Chem. Phys. 93, 1061 (1990).

9 D.E. Makarov, H. Metiu,"Quantum dynamics with dissipation: A treatment of dephasing in the stochastic Schrodinger equation", J. Chem. Phys. 111, 10126 (1999).

10 R. Kapral, G. Ciccotti, "Mixed quantum-classical dynamics", J. Chem. Phys. 110, 8919 (1999).

11 B. Poirier, G. Parlant, "Reconciling semiclassical and Bohmian mechanics: IV. Multisurface Dynamics.", J. Phys. Chem. A 111, 10400 (2007).

12 A. Abedi, G. Agostini, E.K.U. Gross, "Mixed quantum-classical dynamics from exact decomposition of electron-nuclear motion", Europhys. Lett. 106, 33001 (2014).

13 A. McLachlan, "A variational solution of the time-dependent Schrodinger equation", Mol. Phys. 8, 39 (1964).

14 D.A. Micha, J. Chem. Phys. "A self-consistent eikonal treatment of electronic transitions in molecular collisions", 78, 7138 (1983).

15 J. Ren, N. Vukmirovic, L.W. Wang, "Nonadiabatic molecular dynamics simulation for carrier transport in a pentathiophene butyric acid monolayer", Phys, Rev. B 87, 205117 (2013).

16 P.V. Parandekar, J.C. Tully, "Mixed quantum-classical equilibrium", J. Chem. Phys. 122, $094102(2005)$.

17 P.V. Parandekar, "Detailed balance in Ehrenfest mixed quantum-classical dynamics", J.C. Tully, J. Chem. Theory Comput. 2, 229 (2006).

18 C. Zhu, A.W. Jasper, D.G. Truhlar, "Non-Born-Oppenheimer trajectories with self-consistent decay of mixing", J. Chem. Phys. 120, 5543 (2004).

19 G. Granucci, M. Persico, "Critical appraisal of the fewest switches algorithm for surface hopping”, J. Chem. Phys. 126, 134114 (2007).

20 T. Nelson, S. Fernandez-Alberti, A.E. Roitberg, S. Tretiak, "Nonadiabatic excited-state molecular dynamics: Treatment of electronic decoherence", J. Chem. Phys. 138, 224111 (2013).

21 B.R. Landry, J. E. Subotnik, "How to recover Marcus theory with fewest switches surface hopping: Add just a touch of decoherence", J. Chem. Phys. 137, 22A513 (2012).

22 H.M. Jaeger, S. Fischer, O.V. Prezhdo, "Decoherence-induced surface hopping", J. Chem. Phys. 137, 22A545 (2012). 
M.J. Bedard-Hearn, R.E. Larsen, B. J. Schwartz, "Mean-field dynamics with stochastic decoherence (MD-SD): A new algorithm for nonadiabatic mixed quantum/classical molecular-dynamics simulations with nuclear-induced decoherence", J. Chem. Phys. 123, 234106 (2005).

24 S. Hammes-Schiffer, J.C. Tully, "Proton transfer in solution: Molecular dynamics with quantum transitions", J. Chem. Phys. 101, 4657 (1994).

25 W. Kohn, L.J. Sham, "Self-consistent equations including exchange and correlation effects", Phys. Rev. 140, A1133 (1965).

26 E. Runge, E.K. U, Gross, "Density-functional theory for time dependent systems", Phys. Rev. Lett. 52, 997 (1984).

27 A.V. Akimov, O.V. Prezhdo, "The PYXAID program for Non-Adiabatic Molecular Dynamics in Condensed Matter Systems", J. Chem. Theory Comput. 9, 4959 (2013).

28 A.V. Akimov, O.V. Prezhdo, "Advanced capabilities of the PYXAID program: integration schemes, decoherence effects, multiexcitonic states and field-matter interation", J. Chem. Theory Comput. 10, 789 (2014).

29 M. Barbatti, R. Crespo-Otero, "Surface Hopping Dynamics with DFT Excited States", Top. Curr. Chem. doi:10.1007/128_2014_605(2015).

30 E. Tapavicza, I. Tavernelli, U. Rothlisherge, "Trajectory surface hopping within linear response time-dependent density functional theory", Phys. Rev. Lett. 98, 023001 (2007).

31 N.L. Doltsinis, D. Marx, "Nonadiabatic Car-Parrinello molecular dynamics", Phys. Rev. Lett. 88, $166402(2002)$.

32 C.F. Craig, W.R. Duncan, O.V. Prezhdo, "Trajectory surface hopping in the time-dependent Kohn-Sham approach for electron-nuclear dynamics", Phys. Rev. Lett. 95, 163001 (2005).

33 M.S. Schuurman, A. Stolow, "Dynamics at Conical Intersections", Annu. Rev. Phys. Chem. 69, 427 (2018).

34 B.H. Levine, C. Ko, J. Quenneville, T.J. Martinez, "Conical intersections and double excitations in time-dependent density functional theory", Molecular Physics, 104, 1039 (2006).

35 Y. Xiao, Z.W. Wang, L. sHi, X. Jiang, S.S. Li, L.W. Wang, "Anharmonic corrections to the multiphonon deep-level charge capture ab initio calculations for semiconductors", Sci. ChinaPhys. Mech. Astron. 63, 277312 (2020).

36 J. Kang, L.W. Wang "Nonadiabatic molecular dynamics with decoherence and detailed balance under a density matrix assemble formalism", Phys. Rev. B 99, 224303 (2019). 
37 Z. Wang, S.S. Li, L.W. Wang, "An efficient real-time time-dependent DFT method and its applications to ion-2D material collision", Phys. Rev. Lett. 114, 063004 (2015).

38 K.F. Wong, P.J. Rossky, "Solvent-induced electronic decoherence: Configuration dependent dissipative evolution for solvated electron systems", J. Chem. Phys. 116, 8429 (2002).

39 M.R. Sampford, "On sampling without replacement with unequal probabilities of selection", Biometrika, 54, 499 (1967).

40 R.F. Egerton, P. Li, M. Malac, "Radiation damage in the TEM and SEM", Micron 35, 399 (2004).

41 Z. Cai, S. Chen, L.W. Wang, "Dissociation path competition of radiolysis-ionization induced molecule damage under electron beam illumination", Chem. Sci. 10, 10706 (2019).

42 W.H. Liu, J.W. Luo, S.S. Li, L.W. Wang, "Impurity diffusion induced dynamic electron donor in semiconductors", Phys. Rev. B 100, 165203 (2019).

43 C. Lian, S.J. Zhang, S.Q. Hu, M.X. Guan, S. Meng, "Ultrafast charge ordering by self-amplified exciton-phonon dynamics in TiSe2", Nat. Comm. 11, 43 (2020).

44 J. Bang, S. Meng, S.B. Zhang, "Dynamic defect as nonradiative recombination center in semiconductors", Phys. Rev. B 100, 245208 (2019).

45 F. Zheng, L.W. Wang, "Ultrafast hot carrier injection in Au/GaN: the role of band bending and the interface band structure", J. Phys. Chem. Lett. 10, 6174 (2019). 